\title{
Estrés organizacional y salud en funcionarios de centros de atención primaria de una comuna de Santiago
}

\author{
Eliana G uic Sa, Pablo Mora $0^{a}$, Ricardo Rey $C^{b}$, \\ Alfonso Robles G C. \\ Perceived work characteristics \\ and coping strategies that predict \\ distress among w orkers \\ at primary health care centers
} are increasing. The staff perception of specific sources of tension at work is not known. This knowledge is necessary to avoid preventable health problems caused by distress. Aim: To identify specific work stressors in primary health care facilities and to examine individual characteristics that could mediate their effects on health. Material and Methods: We evaluated organizational stressors (Perceived Work Characteristics for Health Services Survey), personal characteristics (Work Locus of Control Scale, Ways of Coping Check List) and distress (Goldberg General Health Questionnaire, Cooper Stress Symptoms Checklist) in a representative sample of 129 workers of 5 public Primary Health Care Centers of a borough in Santiago. Results: Women and nonprofessional personnel reported more psychological and physical stress symptoms. Regression analysis showed that work stressors significantly explained the variance of psychological (26\%) and physical (28\%) distress symptoms. Perception of more role conflicts, less support of peers and superiors and less workload were predictors significantly associated with higher stress outcomes. Avoidance coping strategies were associated with more distress and mediated the effect of organizational stressors on health. Conclusions: We identified the groups at risk of stress in Primary Health Care Centers and specific sources of tension in the organization, as well as individual risk factors. Both should be addressed in interventions to reduce distress and prevent consequences on health (Rev Méd Chile 2006; 134: 447-55).

(Key words: Life change events; Primary health care; Stress, psychological)

\begin{abstract}
Recibido el 20 de julio, 2005. Aceptado el 8 de septiembre, 2005.
aDoctor en Psicología, Escuela de Psicología, Pontificia Universidad Católica de Chile. Santiago, Chile.

bPsicólogo, alumno doctorado, Escuela de Psicología, Pontificia Universidad Católica de Chile. Santiago, Chile.

cAlumno, Escuela de Ingeniería, Pontificia Universidad Católica de Chile. Santiago, Chile.
\end{abstract}

Correspondencia a: Eliana Guic S. Escuela de Psicología, Pontificia Universidad Católica de Chile. Vicuña Mackenna 4860, Santiago, Chile. E mail: eguic@puc.cl 
A unque las condiciones de trabajo, la salud mental y el estrés laboral en funcionarios de salud, han sido motivo de preocupación en Chile desde hace aproximadamente dos décadas ${ }^{1-9}$, no se ha evaluado de forma integral el estrés laboral en la atención primaria. Este nivel de atención cobra cada vez mayor importancia en las políticas actuales en salud. Se le pide, por ejemplo, una mayor capacidad de resolución en un número mayor de problemas médicos, que antes eran de responsabilidad de niveles secundarios. Por esta razón, es de sumo interés conocer la situación de los recursos humanos de este grupo de trabajadores de la salud.

Con el objetivo de abordar el tema en forma integral, en este estudio se usó como marco de referencia el modelo transaccional del estrés propuesto por Lazarus ${ }^{10}$. Este modelo fue aplicado inicialmente al campo del estrés laboral en ejecuti- vos, por Cooper y Bramwell ${ }^{11}$, población que ha sido estudiada con este enfoque en Chile por Guic y cols ${ }^{12}$. En este modelo, se consideran variables del entorno laboral que favorecerían la aparición de reacciones de estrés en los individuos, tanto en salud física como psicológica. Estas respuestas del organismo a situaciones de tensión estarían mediadas por ciertas características de los individuos, que podrían hacerlos más vulnerables al estrés, como las estrategias con que se afrontan las tensiones y la percepción de control sobre los acontecimientos laborales. En la Figura 1 se presenta el modelo teórico, señalando las escalas y subescalas que se utilizaron en este trabajo para evaluar cada una de estas variables. El enfoque transaccional se ha usado previamente para evaluar los diversos factores del estrés en funcionarios de salud en nuestro país. Los trabajos de Trucco y otros autores han sido realizados en organizaciones privadas de salud

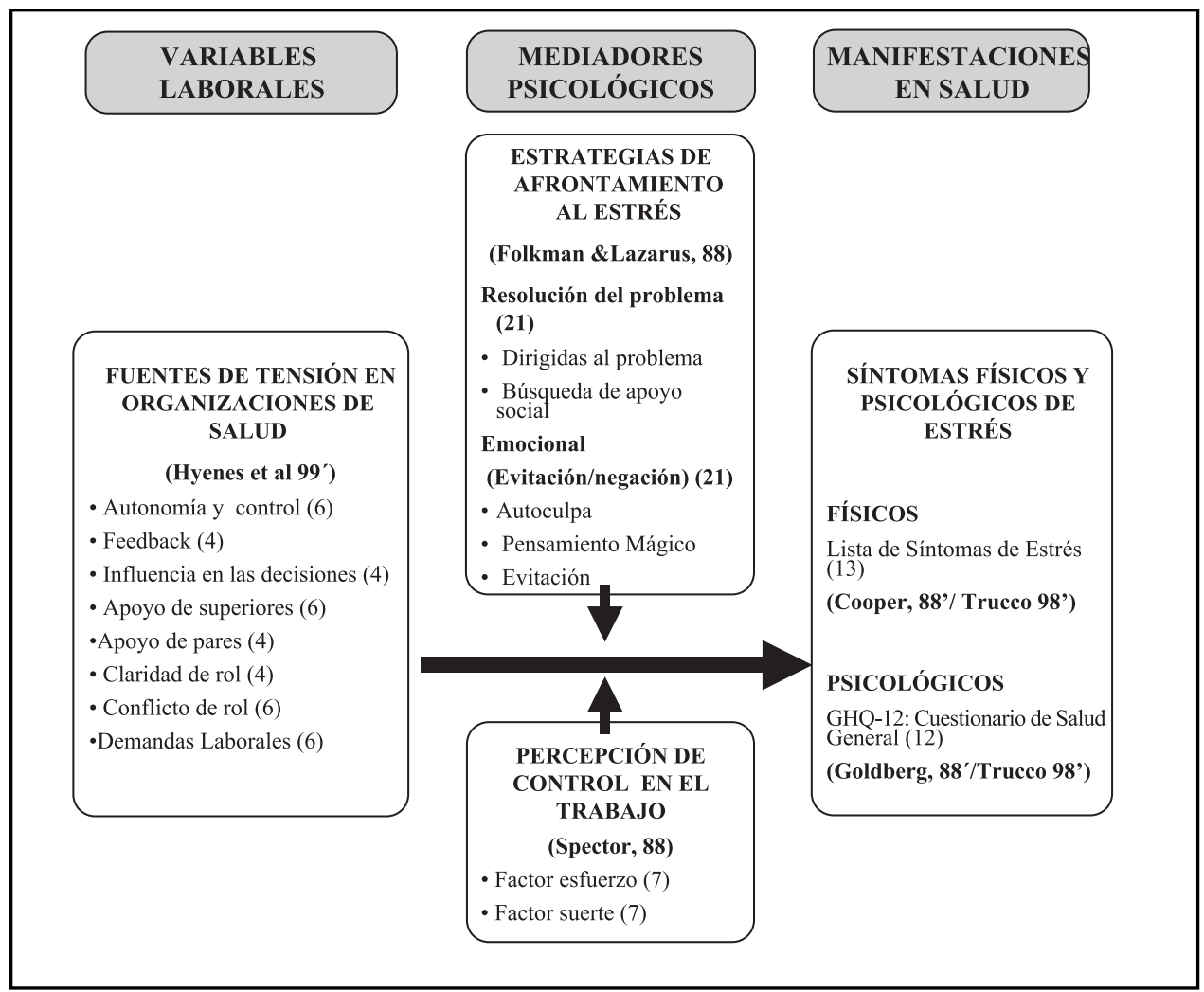

Figura 1. Se presentan las escalas y subescalas utilizadas en el presente trabajo para evaluar, a través de autorreporte, a los funcionarios de salud, en el contexto del modelo transaccional del estrés (ver texto). Se midieron fuentes laborales de tensión, características individuales y manifestaciones del estrés en la salud. Los números entre paréntesis indican la cantidad de ítems en cada subescala. 
0 en el nivel terciario de atención ${ }^{1-3,7,9}$. La única evaluación que se ha reportado en Chile en atención primaria, realizada por Vicente y $\operatorname{cols}^{6}$, evaluó los efectos sobre la salud de los funcionarios, pero sin incluir aspectos laborales.

Los objetivos del presente trabajo son identificar los estresores laborales específicos que predicen síntomas de estrés y evaluar si este efecto en la salud es mediado por características psicológicas individuales, en una muestra representativa de funcionarios, profesionales y no profesionales, de todos los centros de salud de atención primaria, de tipo público, en una comuna de la Región Metropolitana.

\section{Material y MÉTodo}

Muestra y Procedimiento. El tamaño total de la población de funcionarios de los centros de salud de la comuna estudiada era de 506 personas al momento de realizar la evaluación. Este grupo estaba compuesto por médicos, otros profesionales de la salud, y funcionarios no profesionales, entre los que se encuentra el personal administrativo y auxiliares de enfermería. Se tomó una muestra al azar de $30 \%$ de los funcionarios de cada establecimiento, manteniendo las proporciones por sexo y estamento. Se contó con la cooperación de la mayoría de los 158 seleccionados en el muestreo, los resultados presentados se refieren a una muestra de 129 funcionarios. Los restantes 29 no se presentaron o eligieron no responder los cuestionarios una vez informados. Los participantes contestaron los cuestionarios después de leer los objetivos y características del trabajo en el que participaban, de su carácter anónimo, consultar dudas y firmar el consentimiento informado.

Instrumentos. Se utilizaron cinco cuestionarios para evaluar las variables que interesaba estudiar, los que están organizados en tres secciones.

a. Estresores. Se midió la percepción de fuentes de tensión en el trabajo, utilizando la escala Perceived Work Characteristics for Health Services Survey, de 37 ítems de Heynes, Wall, Bolden, Stride y Rick $^{13}$, construida especialmente para organizaciones de salud. El cuestionario original fue traducido y adaptado preliminarmente. El puntaje total de este instrumento comelacionó alta y significativamente $(r=0,78 ; p<0,01)$ con el cuestionario de estresores ocupacionales utilizado, en funcionarios de salud, por Trucco ${ }^{2}$. Esto es una evidencia más de la validez del instrumento utilizado. El rango de la confiabilidad de estas subescalas estuvo entre 0,66 y 0,94.

b. Características personales. Evaluamos percepción de control sobre los acontecimientos laborales y estrategias de afrontamiento orientadas a la resolución de problemas en el trabajo. La variable locus de control fue evaluada con la escala Work Locus of Control Scale, de Spector ${ }^{14}$, que mide el grado en que la persona atribuye a su propio esfuerzo o a factores externos (e.g., suerte) las circunstancias laborales que vive. La versión utilizada fue traducida al español por nosotros y consiste de dos factores que miden los aspectos mencionados (confiabilidad: esfuerzo $=0,64$; suerte $=0,75$ ). La medición de estrategias de afrontamiento fue hecha con la Ways of Coping Check List, de Folkman y Lazarus ${ }^{15}$. Este instrumento contiene 42 ítems que miden la tendencia de las personas a enfocarse en la tarea (21 ítems, confiabilidad $=0,82$ ) 0 en la emoción (21 ítems, confiabilidad $=0,83$ ) al enfrentar un problema.

c. Manifestaciones del estrés. Se midieron síntomas físicos y síntomas psicológicos asociados al estrés.

c.1.Estrés psicológico. Se usó el General Health Questionnaire (GHQ-12) de Goldberg ${ }^{16}$ compuesto por 12 ítems. Trucco y cols ${ }^{1,2}$, describen una adecuada confiabilidad y validez del instrumento traducido, en una muestra de funcionarios de salud y proponen un punto de corte de cuatro puntos para diferenciar entre caso y control. Un puntaje de 4 o más indicaría la existencia de un número de síntomas que permite predecir alta vulnerabilidad a cuadros de ansiedad o depresión.

c.2. Síntomas físicos asociados a estrés. Se utilizó la escala Cooper Stress Symptoms Checklist de 13 ítems $^{17}$, traducida por Trucco y cols ${ }^{2}$ y modificada, eliminando los síntomas psicológicos. No hay datos previos en Chile sobre el punto de corte entre casos y controles en esta escala. Hemos propuesto un puntaje de 12, el que representa el reporte de tres síntomas con una intensidad alta y una frecuencia diaria, lo cual constituiría un indicador clínico de presencia de estrés, según los autores. Al observar cualitativamente los resultados ex- 
puestos en las Figuras 2 y 3, vemos que los promedios que están sobre 12 , remedan a los detectados por el GHQ-12, instrumento bien estudiado en nuestro país, por lo que este punto de corte parece discriminar bien a grupos con o sin estrés. La correlación entre esta escala y la de Goldberg fue moderadaalta $(r=0,56 ; p<0,01)$. Aunque se requiere más estudio psicométrico de esta escala, nuestros datos son una evidencia de la validez del instrumento utilizado.

\section{Resultados}

Se examinó cómo se distribuían los síntomas asociados con estrés (estrés psicológico, síntomas físicos) entre los participantes, cuando éstos se dividían en grupos formados por estamento (médicos, otros profesionales y no profesionales) y por sexo, en los distintos establecimientos (Figuras 2 y 3 ).

Se utilizó análisis de varianza para estudiar diferencias entre grupos. Los resultados para los modelos, utilizando establecimiento y estamento como predictores, mostraron que para la escala de Cooper, sólo la variable estamento se asoció significativamente con síntomas físicos de estrés, $F(2,115)=5,83, p<01$. En análisis post-hoc la única diferencia significativa se encontró entre el grupo de los médicos y el de los no profesionales (t (Scheffé) $=7,10, \mathrm{p}<, 01$ ), teniendo estos últimos mayor sintomatología física de estrés.

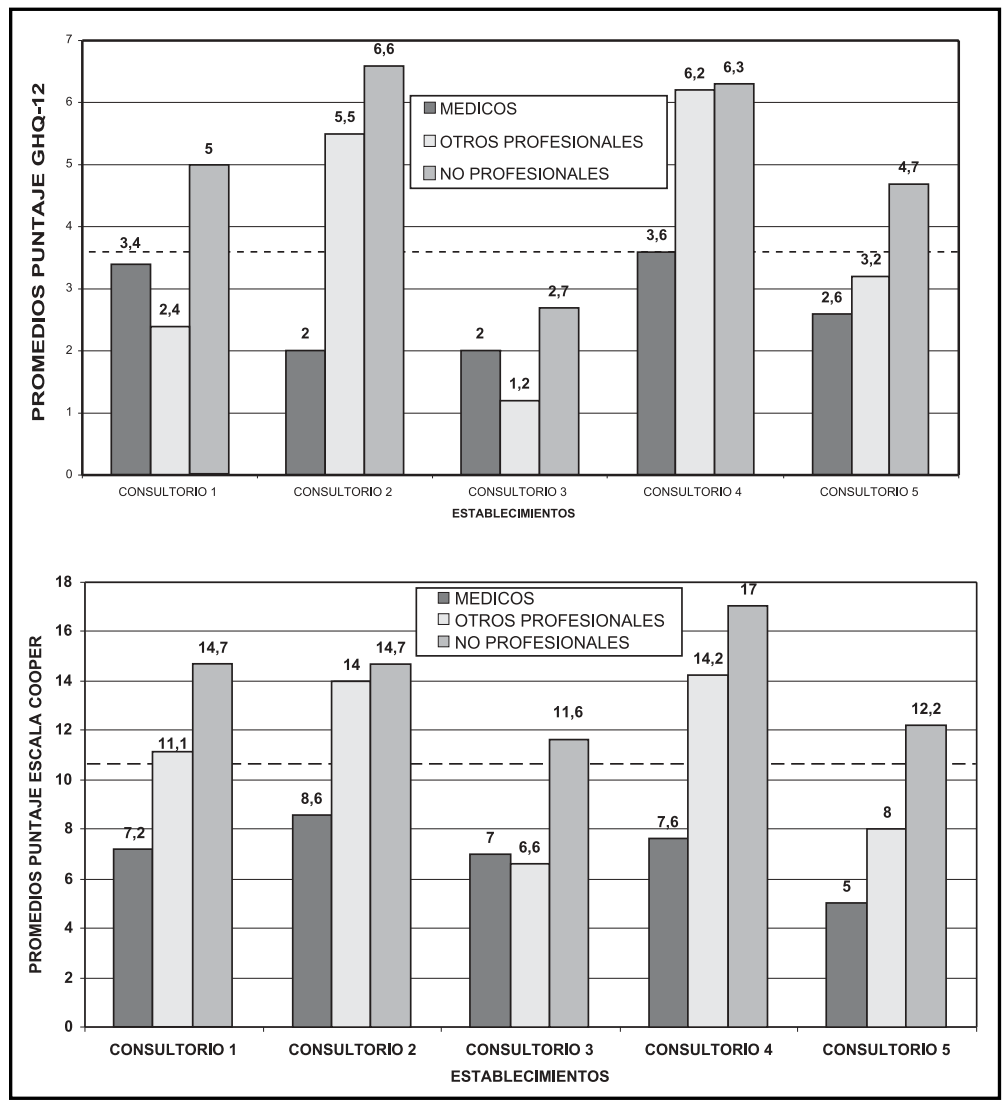

A. Estrés psicológico. Puntaje GHQ-12

B. Síntomas físicos de estrés. Puntaje Escala Cooper.

Figura 2. A. Se presentan datos sobre la presencia de estrés psicológico en la muestra $(\mathrm{N}=129)$. Las barras muestran los promedios en la escala GHQ-12, según estamento y establecimiento. La línea punteada indica el punto de corte entre casos y control $(\mathrm{N}=129)$. B. Se presentan datos sobre síntomas físicos de estrés en la muestra $(\mathrm{N}=129)$. Las barras muestran los promedios en la escala de Síntomas Físicos de Estrés de Cooper, según estamento y establecimiento. La línea punteada indica el punto de corte propuesto para diferenciar entre casos y control $(\mathrm{N}=129)$. 


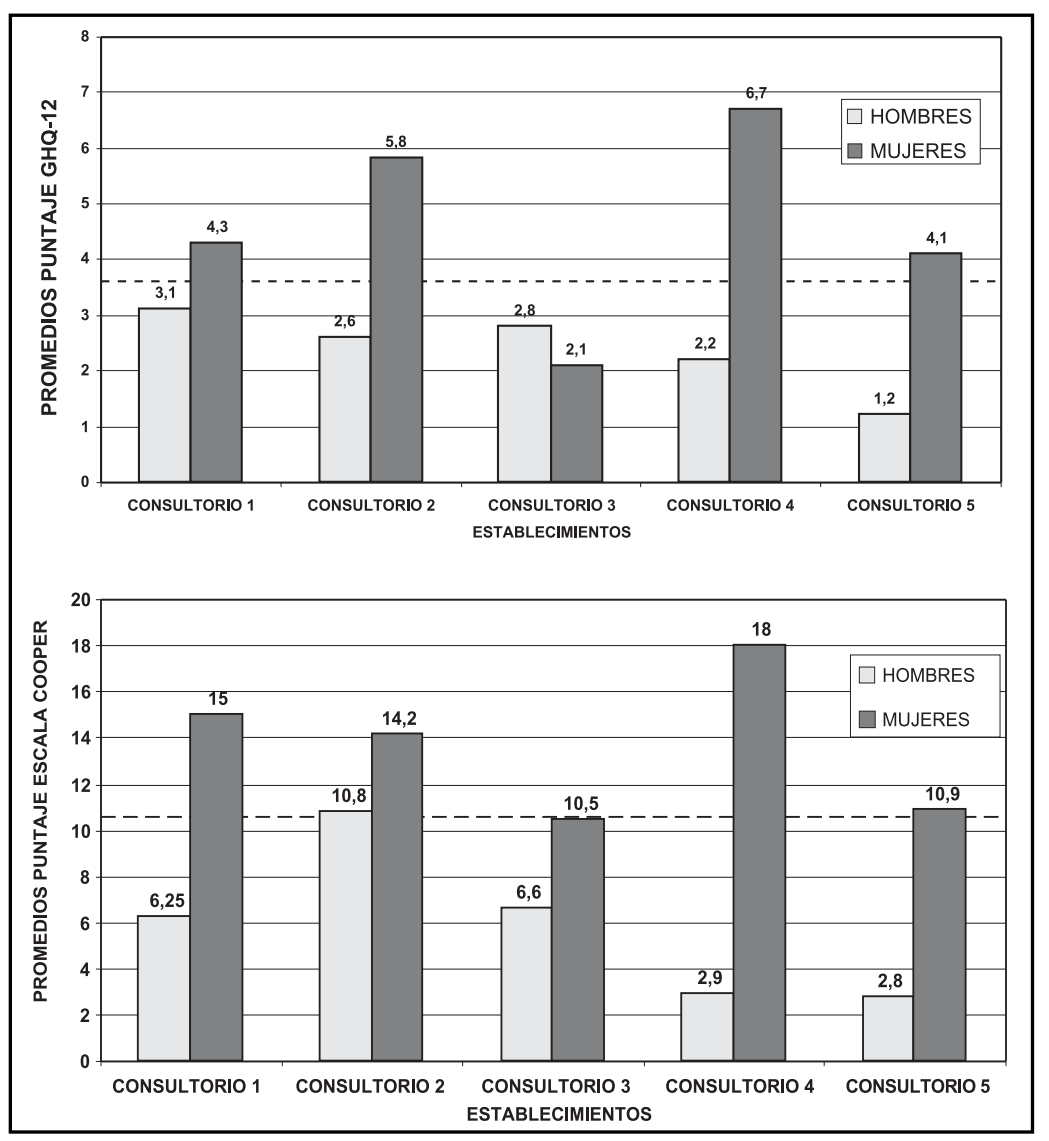

A. Estrés psicológico. Puntaje GHQ-12

B. Síntomas físicos de estrés. Puntaje Escala Cooper.

Figura 3. A. Se presentan datos sobre la presencia de estrés psicológico en la muestra ( $N=129)$. Las barras muestran los promedios en la escala GHQ-12, según sexo y establecimiento. La línea punteada indica el punto de corte entre casos y control. B. Se presentan datos sobre síntomas físicos de estrés en la muestra $(\mathrm{N}=129)$. Las barras muestran los promedios en la escala de Síntomas Físicos de Estrés de Cooper, según sexo y establecimiento. La línea punteada indica el punto de corte propuesto para diferenciar entre casos y control $(\mathrm{N}=129)$.

De forma similar, la variable estamento se asoció con síntomas de estrés emocional, aunque marginalmente $(F(2,115)=2,93, p=, 057)$, mientras que establecimiento no mostró ninguna asociación. Los análisis post-hoc mostraron que este resultado se debió principalmente a las diferencias entre el estamento médico y el no profesional. En ninguno de los casos las interacciones entre estamento y establecimiento fueron significativas. Un análisis descriptivo de los datos mostró que el grupo estamento médico no superó el punto de corte en la escala de Goldberg en ninguno de los centros de salud, mientras que los grupos no profesionales presentaron puntajes promedio en estrés emocional mayores que el puntaje de corte en los establecimientos 1, 2, 4 y 5. Los síntomas físicos de estrés mostraron la misma tendencia (Figura 2 A y B).

Como muestran las Figuras 3 A y $\mathrm{B}$, en general, las mujeres mostraron más síntomas de estrés emocional $(F(1,120)=6,28, p<05)$, como también de síntomas físicos $(F(1,120)=14,78, p$ $<, 01)$. Ni la variable establecimiento ni la interacción entre variables estuvieron significativamente asociadas con las variables dependientes. En los gráficos se observa que las mujeres sobrepasaron los límites de corte para síntomas de estrés emocional en tres establecimientos. Los hombres 
Tabla 1. Betas y cambios en la varianza explicada $\left(\Delta \mathbf{R}^{2}\right)$ de modelos de regresión prediciendo síntomas de estrés emocional y síntomas físicos de estrés con estresores laborales

\begin{tabular}{|c|c|c|}
\hline & $\begin{array}{l}\text { Estrés emocional } \\
\text { Beta final }\end{array}$ & $\begin{array}{l}\text { S físicos de estrés } \\
\text { Beta final }\end{array}$ \\
\hline \multicolumn{3}{|l|}{ Variables control } \\
\hline Sexo & 08 & ,18* \\
\hline Estamento &,- 09 &, $17 *$ \\
\hline$\Delta \mathrm{R}^{2}$ & $0,06^{*}$ & $0,12^{* *}$ \\
\hline \multicolumn{3}{|l|}{ Estresores laborales } \\
\hline Demandas laborales &,- 06 &,$- 29 * *$ \\
\hline Autonomía-control &,- 15 &,- 17 \\
\hline Feedback & ,01 &, 03 \\
\hline Influencia en decisiones &, 08 &,- 04 \\
\hline Apoyo de superiores &,- 20 &,$- 28 *$ \\
\hline Claridad de rol &, 01 & 17 \\
\hline Conflicto de rol & $29 * *$ &,- 13 \\
\hline Apoyo de pares &,$- 19 *$ &,- 04 \\
\hline$\Delta \mathrm{R}^{2}$ & 0,26 ** & 0,28 ** \\
\hline
\end{tabular}

*p $<0,05 ; *$ * $<0,001$.

no sobrepasaron los puntajes de corte en ninguno (Figura 3A). Los síntomas físicos de estrés mostraron una tendencia similar (Figura 3B).

Identificación de los predictores laborales de síntomas emocionales y físicos de estrés. Para determinar cuáles estresores ocupacionales se mostraban como mejores predictores de los síntomas de estrés emocional y síntomas físicos de estrés, se usó análisis de regresión múltiple (Tabla 1).

Cuando se modelaron las diferencias individuales en estrés emocional, los resultados mostraron que los únicos estresores que predijeron esta variable significativamente fueron conflicto de rol y apoyo de pares. Esto significa que mayor conflicto de rol y menor apoyo de pares se asociaron con más síntomas de estrés emocional. Es importante señalar que otros dos estresores laborales, autonomía-control (menor autonomía) y apoyo de superiores (menor apoyo) se relacionaron con mayores niveles de estrés emocional en forma marginalmente significativa. A pesar que solamente dos de los estresores mostraron asociaciones significativas, el conjunto de ellos logra explicar un porcentaje de la varianza considerado alto en este tipo de estudios (26\%).

Los análisis para síntomas físicos de estrés mostraron que además de las variables de control, sexo y estamento (ser mujer y no profesional), las variables demandas laborales y apoyo de superiores se asociaron significativamente con síntomas físicos de estrés. Estos resultados indican que la percepción de menores demandas laborales y de menor apoyo de superiores se relacionan con el reporte de más síntomas físicos de estrés. Además de las variables ya mencionadas, autonomía-control (menor autonomía) y claridad de rol (menor claridad de rol) se asociaron marginalmente con síntomas físicos de estrés $(p<0,052)$. En este análisis, los estresores en su conjunto explicaron una proporción de la varianza alta y significativa (28\%), similar a la explicada para estrés emocional.

Rol mediador de características individuales en el efecto de estresores laborales sobre síntomas emocionales y físicos de estrés. Se examinó si las variables personales medidas (tipo de afrontamiento al estrés y locus de control) mediaban el 
Tabla 2. Betas y cambios en la varianza explicada $\left(\Delta \mathbf{R}^{2}\right)$ de modelos de regresión examinando efectos mediadores de locus de control y afrontamiento

\begin{tabular}{|c|c|c|c|c|}
\hline & \multicolumn{2}{|c|}{ Estrés emocional } & \multicolumn{2}{|c|}{ S físicos de estrés } \\
\hline & Modelo 1 & Modelo 2 & Modelo 1 & Modelo 2 \\
\hline \multicolumn{5}{|l|}{ Variables control } \\
\hline Sexo & 0,10 & 0,11 & $0,22 *$ & $0,23^{* *}$ \\
\hline Estamento & $-0,05$ & $-0,01$ & $-0,16$ & $-0,14$ \\
\hline$\Delta \mathrm{R}^{2}$ & 0,05 & & 0,13 & \\
\hline \multicolumn{5}{|l|}{ Estresores laborales } \\
\hline Demandas laborales & $-0,06$ & 0,03 & $-0,23^{* *}$ & $-0,15$ \\
\hline Autonomía-control & $-0,13$ & 0,02 & $-0,16$ & $-0,03$ \\
\hline Apoyo de superiores & $-0,16$ & $-0,07$ & $-0,26$ ** & $-0,18^{*}$ \\
\hline Conflicto de rol & $0,25^{\text {** }}$ & $-0,20 *$ & $-0,04$ & 0,02 \\
\hline Apoyo de pares & $-0,19 *$ & $-0,18^{*}$ & $-0,03$ & $-0,02$ \\
\hline$\Delta \mathrm{R}^{2}$ & 0,24 & & 0,21 & \\
\hline \multicolumn{5}{|l|}{ Mediadores } \\
\hline Locus de control (suerte) & &,- 15 & &,- 11 \\
\hline Locus de control (esfuerzo) & & 0,05 & & 0,01 \\
\hline Afrontamiento problema & & $-0,07$ & & 0,00 \\
\hline Afrontamiento emoción & & $0,48^{\text {** }}$ & & 0,46 ** \\
\hline$\Delta \mathrm{R}^{2}$ & 0,20 & & 0,19 & \\
\hline
\end{tabular}

$*_{p}<0,05 ; *^{*}<<0,001$.

efecto de los estresores en los síntomas de estrés emocional y síntomas físicos de estrés, como lo propone el modelo transaccional. Para esto analizamos si los efectos de los estresores que mostraron asociación significativa con ambas medidas de estrés (Modelos 1 en Tabla 2) estaban mediados por las variables personales (Modelos 2 en Tabla 2). Para facilitar la comparación se utilizaron las mismas variables para cada modelo.

Los resultados para estrés emocional (Modelo 1) mostraron que conflicto de rol y apoyo de pares se mantuvieron significativamente asociadas con síntomas de estrés emocional. Apoyo de superiores se asoció marginalmente con estrés emocional. Cuando las variables mediadores fueron entradas en el modelo (Modelo 2), la fuerza de estas asociaciones se redujo, aunque levemente. Es importante señalar que de las características psicológicas evaluadas, solamente afrontamiento de evitación se asoció con más síntomas de estrés emocional. Dados estos resultados, es posible decir que esta variable media parcialmente los efectos de los estresores en estrés emocional. Locus de control no mostró ningún tipo de asociación con síntomas de estrés emocional.

Los resultados para síntomas físicos de estrés fueron similares a los de estrés emocional. En este caso, afrontamiento orientado a la emoción mostró un efecto mediador comparativamente mayor. La otra variable mediadora (locus de control), tal como sucedió en el caso de estrés emocional, no mostró ninguna asociación con síntomas físicos de estrés. Es importante recalcar que los efectos de sexo se mantuvieron significativos y de tamaño moderado.

\section{Discusión}

Los datos obtenidos en una muestra representativa de funcionarios de establecimientos públicos de 
atención primaria de una comuna de Santiago, mostraron que las mujeres y los funcionarios no profesionales presentan puntajes significativamente mayores en las escalas de síntomas de estrés emocional y estrés físico. Es importante recordar que las funcionarias mujeres no profesionales constituyen el grupo más grande dentro de los funcionarios de salud.

Además de identificar al grupo de mayor riesgo, nuestro trabajo identificó estresores laborales propios de organizaciones de salud a nivel de atención primaria. Los predictores significativos de síntomas psicológicos o físicos de estrés incluyeron: la presencia de un mayor conflicto de rol, menor apoyo de pares y de superiores, así como también, interesantemente, una menor demanda laboral. Fueron marginalmente significativos autonomía en el trabajo y claridad de rol. Todas estas características organizacionales han sido descritas previamente como posibles estresores laborales ${ }^{9,18-20}$. Nuestros resultados muestran asociaciones en las direcciones esperadas, excepto por demandas laborales. La dirección de esta asociación normalmente es opuesta a la descrita en el presente trabajo ${ }^{19,20}$, sin embargo, también se ha reportado que una percepción de subcarga laboral puede contribuir al estrés laboral ${ }^{18}$.

Es posible proponer otra interpretación para este resultado, la que debiera ser estudiada con mayor detalle en el futuro. Habría que diferenciar si es realmente una subcarga laboral lo que está siendo estresante, o si el estrés aumenta porque las personas se perciben como que hacen poco para resolver los problemas de salud y se sienten no productivas en el trabajo. Esto último significaría que los datos no reflejan necesariamente que la carga de trabajo es baja. Se puede aventurar que lo que existe es una sensación subjetiva de que no se logra hacer lo suficiente para mejorar la

\section{REFERENCIAS}

1. Trucco M, Campusano ME, LarRaín S. Un cuestionario para detectar desórdenes emocionales: estudio de validación preliminar. Rev Chil Neuro-Psiquiat 1979; 17: 20-5. salud de las personas atendidas. Esto sería coherente con una percepción de falta de autonomía y control en el trabajo, que mostró una tendencia a predecir alto estrés en la muestra estudiada.

Nuestros datos confirman la propuesta teórica de que hay características personales relativamente persistentes, como estilos de afrontamiento, que median parcialmente el efecto de los estresores sobre la salud física y psicológica. Se observó que las personas que tienden a usar estrategias de evitación o negación de los problemas que se le presentan en el trabajo, tienen mayores niveles de estrés. La negación o evitación del problema permite a las personas reducir su síntomas de estrés, pero sin cambiar los factores que son la causa de ellos. Esto permite un mejor funcionamiento psicológico y son de gran importancia adaptativa, sobre todo durante el primer tiempo. Pero finalmente, la negación puede generar mayor estrés porque no se logra resolver los problemas, lo que puede llevar a una reacción en espiral. Esto implica que el uso de estrategias orientadas a buscar información, pedir ayuda y promover cambios que intenten resolver los conflictos, tienen un efecto positivo en la reducción del estrés laboral.

En resumen, nuestros resultados muestran que para abordar adecuadamente el problema del estrés en los establecimientos de salud, es importante evaluar no sólo factores organizacionales sino también personales, ya que los factores individuales ejercen un efecto mediador sobre el estrés. Sin embargo, los resultados también sugieren que una intervención basada solamente sobre aspectos personales no permitiría una reducción significativa de los niveles de estrés en las personas, ya que la mediación es parcial. En general, los resultados confirman lo descrito previamente para otro tipo de organizaciones laborales y en términos teónicos validan el modelo transaccional del estrés ${ }^{18,21-26}$.

2. TRucco M, Valenzuela P. Una batería de cuestionarios para el estudio de estrés ocupacional. Rev Chil Neuro-Psiquiat 1998; 36: 159-65.

3. Trucco M, Valenzuela P, Trucco D. Estrés Ocupacional en Personal de Salud. Rev Méd Chile 1999; 127: 1453-61. 
4. Ibáñez J, Miguel H, Martínez O, Gacitúa S. Riesgo laboral y patología psiquiátrica del personal de un servicio de Psiquiatría. Rev Chil Neuro-Psiquiat 1988; 26: 123-32.

5. Vega MS, Osorio R. Detección de Factores de Stress Laboral y Extralaboral en Funcionarios del Hospital Psiquiátrico de Santiago. Revista de Psiquiatría 1985; 2: 287-92.

6. Vicente B, Saldivia S, Rioseco P, Vielma M, Escobar B, Medina E. Niveles de salud mental de los funcionarios de dos consultorios generales urbanos. Revista de Psiquiatría 1992; 9: 159-65.

7. Avendaño C, Leiva P. Riegos para la salud psicológica en enfermeras. Psykhe 1998; 7(2).

8. HeRnández H, EscRibano I. Estrés Laboral y Relaciones Interpersonales: Sistematización de la Experiencia en el Área de Estrés Laboral. Informe del Servicio de Salud Concepción-Arauco, 1995.

9. Landa V, Mena L Estrés Laboral en personal clínico hospitalario. Terapia Psicológica 2003; 21: 137-51.

10. LAzARus R. Stress and Emotion. London: Free Association Books, 1999.

11. Cooper CL, Bramwell R. Predictive validity of the strain components of the occupational stress indicator. Stress Med 1992; 8: 56-60.

12. Guic E, Bilbao R, Bertin C. Estrés laboral y salud en una muestra de ejecutivos chilenos. Rev Méd Chile 2002; 130: 1101-12.

13. Heynes C, Wall T, Bolden R, Stride C, Rick J. Measures of perceived work characteristics for health services research: Test of a measurement model and normative data. Br J Health Psychol 1999; 4: 257-75.

14. Spector PE. Development of the Work Locus of Control Scale. Journal of Occupational Psychology 1988; 61: 335-40.

15. FolKman S, Lazarus RS. Coping as a mediator of emotion. J Personal and Social Psychol 1988; 54: 466-75.

16. GoLDBerg D, WiLAMs P. Windsory Berkshire ed. A user's guide to the General Health Questionnaire. NFER-NELSON, 1988.
17. COOPER C. The stress check: coping with stress of life and work. Englewood Cliffs NJ, Ed. PrenticeHall, Inc., 1981.

18. Wiшамs S. Ways of Creating Healthy Work Organizations. En: Cooper C L and Williams S, eds. Creating Healthy Work Organizations. Chichester: John Wiley \& Sons, 1994: 7-24.

19. GRIFFin M, HaRT P, WiLSON-Evered E. Using employee opinion surveys to improve organizational health. En: L. Murphy y C.L. Cooper, eds. Healthy and Productive Work. London: Taylor \& Francis, 2000: 15-36.

20. Fingret A. Developing a Company Mental Health Plan. En: Cooper C. L. y Williams S. eds. Creating Healthy Work Organizations. Chichester: John Wiley \& Sons, 1994: 97-114.

21. Judge CH. An Organisational Stress Audit: BNR Europe. En: Cooper C. L. y Williams S. eds. Creating Healthy Work Organizations. Chichester: John Wiley \& Sons, 1994: 115-32.

22. Lfakey P, Littlewood M, Reynolds S, Bunce D. Caring for the Carers: North Derbyshire Health Authority. En: Cooper C. L. y Williams S, eds. Creating Healthy Work Organizations. Chichester: John Wiley \& Sons, 1994: 167-96.

23. Muier D. Gaining Control over the Work Environment. En: Cooper C. L y Williams S, eds. Creating Healthy Work Organizations. Chichester: John Wiley \& Sons, 1994: 197-214.

24. SAUTER S, Lim S, MuRPHY L. Organizational health: A new paradigm for occupational stress research at NIOSH. Japanese Journal of Occupational Mental Health 1996; 4: 248-54.

25. Murphy L, Cooper C. Models of healthy work organizations. En: Murphy L y Cooper C. L, eds. Healthy and Productive Work. London: Taylor \& Francis, 2000: 1-11.

26. Moran S, PARRY T. Managing change in healthcare: a study of one hospital system's efforts. En: Murphy L. y Cooper C. L, eds. Healthy and Productive Work. London: Taylor \& Francis, 2000: 51-65.

Agradecimientos

A la Escuela de Psicología de la Pontificia Universidad Católica de Chile por financiar parcialmente este trabajo. 